\title{
A hypothetical upper bound for the solutions of a Diophantine equation with a finite number of solutions
}

\section{Apoloniusz Tyszka}

\begin{abstract}
We conjecture that if a system $S \subseteq\left\{x_{i}=1, x_{i}+x_{j}=x_{k}, x_{i} \cdot x_{j}=x_{k}\right.$ : $i, j, k \in\{1, \ldots, n\}\}$ has only finitely many solutions in integers $x_{1}, \ldots, x_{n}$, then each such solution $\left(x_{1}, \ldots, x_{n}\right)$ satisfies $\left|x_{1}\right|, \ldots,\left|x_{n}\right| \leq 2^{2^{n-1}}$. By the conjecture, if a Diophantine equation has only finitely many solutions in integers (non-negative integers, rationals), then their heights are bounded from above by a computable function of the degree and the coefficients of the equation. The conjecture implies that the set of Diophantine equations which have infinitely many solutions in integers (non-negative integers) is recursively enumerable. The conjecture formulated for an arbitrary computable bound instead of $2^{2^{n-1}}$ remains in contradiction to Matiyasevich's conjecture that each recursively enumerable set $\mathcal{M} \subseteq \mathbb{N}^{n}$ has a finite-fold Diophantine representation.
\end{abstract}

2010 Mathematics Subject Classification: 03D20, 11 U05.

Key words and phrases: computable upper bound for the heights of integer (rational) solutions of a Diophantine equation, Davis-Putnam-RobinsonMatiyasevich theorem, Diophantine equation with a finite number of integer (rational) solutions, single-fold (finite-fold) Diophantine representation, system of Diophantine equations.

The Davis-Putnam-Robinson-Matiyasevich theorem states that every recursively enumerable set $\mathcal{M} \subseteq \mathbb{N}^{n}$ has a Diophantine representation, that is

$$
\left(a_{1}, \ldots, a_{n}\right) \in \mathcal{M} \Longleftrightarrow \exists x_{1}, \ldots, x_{m} \in \mathbb{N} W\left(a_{1}, \ldots, a_{n}, x_{1}, \ldots, x_{m}\right)=0
$$

for some polynomial $W$ with integer coefficients, see [8] and [7]. The polynomial $W$ can be computed, if we know a Turing machine $M$ such that, for all $\left(a_{1}, \ldots, a_{n}\right) \in \mathbb{N}^{n}, M$ halts on $\left(a_{1}, \ldots, a_{n}\right)$ if and only if $\left(a_{1}, \ldots, a_{n}\right) \in \mathcal{M}$, see [8] and [7]. The representation ( $\mathrm{R}$ ) is said to be finite-fold if for any $a_{1}, \ldots, a_{n} \in \mathbb{N}$ the equation $W\left(a_{1}, \ldots, a_{n}, x_{1}, \ldots, x_{m}\right)=0$ has at most finitely many solutions $\left(x_{1}, \ldots, x_{m}\right) \in \mathbb{N}^{m}$. Yu. Matiyasevich conjectures that each recursively enumerable set $\mathcal{M} \subseteq \mathbb{N}^{n}$ has a finite-fold Diophantine representation, see [4, pp. 341-342], [9, p. 42] and [10, p. 79]. His conjecture remains in contradiction to the following Conjecture, see Corollary 2 . 
Conjecture. For each positive integer $n$, if a system

$$
S \subseteq E_{n}=\left\{x_{i}=1, x_{i}+x_{j}=x_{k}, x_{i} \cdot x_{j}=x_{k}: i, j, k \in\{1, \ldots, n\}\right\}
$$

has only finitely many solutions in integers $x_{1}, \ldots, x_{n}$, then each such solution $\left(x_{1}, \ldots, x_{n}\right)$ satisfies $\left|x_{1}\right|, \ldots,\left|x_{n}\right| \leq 2^{2^{n-1}}$.

Let $T_{n}$ denote the set of all integer tuples $\left(a_{1}, \ldots, a_{n}\right)$ for which there exists a system $S \subseteq E_{n}$ such that $\left(a_{1}, \ldots, a_{n}\right)$ solves $S$ and $S$ has at most finitely many solutions in integers $x_{1}, \ldots, x_{n}$. If $\left(a_{1}, \ldots, a_{n}\right) \in T_{n}$, then $\left(a_{1}, \ldots, a_{n}\right)$ solves the system

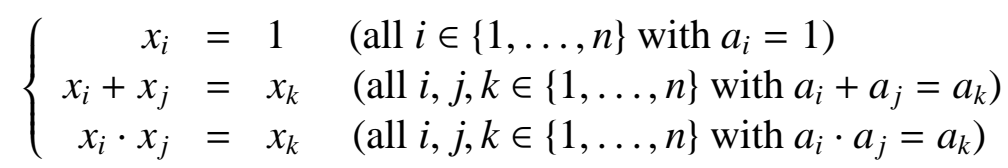

which has only finitely many solutions in integers $x_{1}, \ldots, x_{n}$.

Proposition. The Conjecture is true for $n \leq 3$.

Proof. $T_{1}=\{0,1\} . T_{2}$ consists of the pairs $(0,0),(1,1),(-1,1),(0,1),(1,2),(2,4)$ and their permutations. $T_{3}$ consists of the triples

$$
\begin{gathered}
(0,0,0),(1,1,1), \\
(-1,-1,1),(0,0,1),(1,1,-1),(1,1,0),(1,1,2),(2,2,1),(2,2,4),(4,4,2), \\
(1,-2,-1),(1,-1,0),(1,-1,2),(1,0,2),(1,2,3),(1,2,4), \\
(2,4,-2),(2,4,0),(2,4,6),(2,4,8),(2,4,16), \\
(-4,-2,2),(-2,-1,2),(3,6,9),(4,8,16)
\end{gathered}
$$

and their permutations.

Let $C_{n}$ denote the Conjecture restricted to the systems $S \subseteq E_{n}$.

Lemma 1. For each positive integer $n$, if $C_{n}$ is false then $C_{n+1}$ is false.

Proof. Since $C_{n}$ is false, there exist integers $a_{1}, \ldots, a_{n}$ and a system $S \subseteq E_{n}$ such that $\left(a_{1}, \ldots, a_{n}\right)$ solves $S,\left|a_{n}\right|>2^{2^{n-1}}$, and the system $S$ has only finitely many solutions in integers $x_{1}, \ldots, x_{n}$. Then, $\left|a_{n}^{2}\right|>2^{2^{n}}$ and the integer $(n+1)$-tuple $\left(a_{1}, \ldots, a_{n}, a_{n}^{2}\right)$ solves the system $S \cup\left\{x_{n} \cdot x_{n}=x_{n+1}\right\}$ which has only finitely many solutions in integers $x_{1}, \ldots, x_{n}, x_{n+1}$. 
The Conjecture seems to be true for $\mathbb{N}, \mathbb{N} \backslash\{0\}, \mathbb{Q}, \mathbb{R}$ and $\mathbb{C}$, cf. [20, p. 528, Conjecture 5d], [21, p. 177, Conjecture 1.5(d)] and [21, p. 180]. For $n \geq 2$, the bound $2^{2^{n-1}}$ cannot be decreased because the system

$$
\left\{\begin{array}{rll}
x_{1}+x_{1} & =x_{2} \\
x_{1} \cdot x_{1} & =x_{2} \\
x_{2} \cdot x_{2} & =x_{3} \\
x_{3} \cdot x_{3} & =x_{4} \\
& \cdots \\
x_{n-1} \cdot x_{n-1} & =x_{n}
\end{array}\right.
$$

has precisely two integer solutions, namely $(0, \ldots, 0)$ and $\left(2,4,16,256, \ldots, 2^{2^{n-2}}, 2^{2^{n-1}}\right)$. Nevertheless, for each integer $n \geq 12$ there is a system $S \subseteq E_{n}$ which has infinitely many integer solutions and they all belong to $\mathbb{Z}^{n} \backslash\left[-2^{2^{n-1}}, 2^{2^{n-1}}\right]^{n}$, see [19, p. 4, Theorem 1], cf. [21, p. 178, Theorem 2.4]. The next theorem generalizes this.

Theorem 1. ([19] p. 7, Theorem 2]) There is an algorithm that for every computable function $f: \mathbb{N} \rightarrow \mathbb{N}$ returns a positive integer $m(f)$, for which a second algorithm accepts on the input $f$ and any integer $n \geq m(f)$, and returns a system $S \subseteq E_{n}$ such that $S$ has infinitely many integer solutions and each integer tuple $\left(x_{1}, \ldots, x_{n}\right)$ that solves $S$ satisfies $x_{1}=f(n)$.

Theorem 2. ([19 p. 8, Corollary]) There is an algorithm that for every computable function $f: \mathbb{N} \rightarrow \mathbb{N}$ returns a positive integer $m(f)$, for which a second algorithm accepts on the input $f$ and any integer $n \geq m(f)$, and returns an integer tuple $\left(x_{1}, \ldots, x_{n}\right)$ for which $x_{1}=f(n)$ and

(1) for each integers $y_{1}, \ldots, y_{n}$ the conjunction

$$
\begin{gathered}
\left(\forall i \in\{1, \ldots, n\}\left(x_{i}=1 \Longrightarrow y_{i}=1\right)\right) \wedge \\
\left(\forall i, j, k \in\{1, \ldots, n\}\left(x_{i}+x_{j}=x_{k} \Longrightarrow y_{i}+y_{j}=y_{k}\right)\right) \wedge \\
\forall i, j, k \in\{1, \ldots, n\}\left(x_{i} \cdot x_{j}=x_{k} \Longrightarrow y_{i} \cdot y_{j}=y_{k}\right)
\end{gathered}
$$

implies that $x_{1}=y_{1}$.

If $n \geq 2$, then the tuple $\left(x_{1}, \ldots, x_{n}\right)=\left(2^{2^{n-2}}, 2^{2^{n-3}}, \ldots, 256,16,4,2,1\right)$ has property (1). Unfortunately, we do not know any explicitly given integers $x_{1}, \ldots, x_{n}$ with property (1) and $\left|x_{1}\right|>2^{2^{n-2}}$. 
To each system $S \subseteq E_{n}$ we assign the system $\widetilde{S}$ defined by

$$
\begin{gathered}
\left(S \backslash\left\{x_{i}=1: i \in\{1, \ldots, n\}\right\}\right) \cup \\
\left\{x_{i} \cdot x_{j}=x_{j}: i, j \in\{1, \ldots, n\} \text { and the equation } x_{i}=1 \text { belongs to } S\right\}
\end{gathered}
$$

In other words, in order to obtain $\widetilde{S}$ we remove from $S$ each equation $x_{i}=1$ and replace it by the following $n$ equations:

$$
\begin{aligned}
x_{i} \cdot x_{1} & =x_{1} \\
& \ldots \\
x_{i} \cdot x_{n} & =x_{n}
\end{aligned}
$$

Lemma 2. For each system $S \subseteq E_{n}$

$$
\begin{array}{r}
\left\{\left(x_{1}, \ldots, x_{n}\right) \in \mathbb{Z}^{n}:\left(x_{1}, \ldots, x_{n}\right) \text { solves } \widetilde{S}\right\} \\
\left\{\left(x_{1}, \ldots, x_{n}\right) \in \mathbb{Z}^{n}:\left(x_{1}, \ldots, x_{n}\right) \text { solves } S\right\} \cup\{(0, \ldots, 0)\}
\end{array}
$$

By Lemma 2, the Conjecture is equivalent to

$$
\begin{gathered}
\forall x_{1}, \ldots, x_{n} \in \mathbb{Z} \exists y_{1}, \ldots, y_{n} \in \mathbb{Z} \\
\left(2^{2^{n-1}}<\left|x_{1}\right| \Longrightarrow\left(\left|x_{1}\right|<\left|y_{1}\right| \vee \ldots \vee\left|x_{1}\right|<\left|y_{n}\right|\right)\right) \wedge \\
\left(\forall i, j, k \in\{1, \ldots, n\}\left(x_{i}+x_{j}=x_{k} \Longrightarrow y_{i}+y_{j}=y_{k}\right)\right) \wedge \\
\forall i, j, k \in\{1, \ldots, n\}\left(x_{i} \cdot x_{j}=x_{k} \Longrightarrow y_{i} \cdot y_{j}=y_{k}\right)
\end{gathered}
$$

The statement

$$
\begin{gathered}
\forall x_{1}, \ldots, x_{n} \in \mathbb{Z} \exists y_{1}, \ldots, y_{n} \in \mathbb{Z} \\
\left(2^{2^{n-1}}<\left|x_{1}\right| \Longrightarrow\left|x_{1}\right|<\left|y_{1}\right|\right) \wedge \\
\left(\forall i, j, k \in\{1, \ldots, n\}\left(x_{i}+x_{j}=x_{k} \Longrightarrow y_{i}+y_{j}=y_{k}\right)\right) \wedge \\
\forall i, j, k \in\{1, \ldots, n\}\left(x_{i} \cdot x_{j}=x_{k} \Longrightarrow y_{i} \cdot y_{j}=y_{k}\right)
\end{gathered}
$$

obviously strengthens the Conjecture, but is false for some $n$. The last observation follows from Theorem 2 .

Let $D\left(x_{1}, \ldots, x_{p}\right) \in \mathbb{Z}\left[x_{1}, \ldots, x_{p}\right] . \quad$ For the Diophantine equation $2 \cdot D\left(x_{1}, \ldots, x_{p}\right)=0$, let $M$ denote the maximum of the absolute values of its coefficients. Let $\mathcal{T}$ denote the family of all polynomials $W\left(x_{1}, \ldots, x_{p}\right) \in \mathbb{Z}\left[x_{1}, \ldots, x_{p}\right]$ whose all coefficients belong to the interval $[-M, M]$ and $\operatorname{deg}\left(W, x_{i}\right) \leq d_{i}=\operatorname{deg}\left(D, x_{i}\right)$ for each $i \in\{1, \ldots, p\}$. Here we 
consider the degrees of $W\left(x_{1}, \ldots, x_{p}\right)$ and $D\left(x_{1}, \ldots, x_{p}\right)$ with respect to the variable $x_{i}$. It is easy to check that

$$
\operatorname{card}(\mathcal{T})=(2 M+1)^{\left(d_{1}+1\right) \cdot \ldots \cdot\left(d_{p}+1\right)}
$$

We choose any bijection $\tau:\{p+1, \ldots, \operatorname{card}(\mathcal{T})\} \rightarrow \mathcal{T} \backslash\left\{x_{1}, \ldots, x_{p}\right\}$. Let $\mathcal{H}$ denote the family of all equations of the forms

$$
x_{i}=1, x_{i}+x_{j}=x_{k}, x_{i} \cdot x_{j}=x_{k}(i, j, k \in\{1, \ldots, \operatorname{card}(\mathcal{T})\})
$$

which are polynomial identities in $\mathbb{Z}\left[x_{1}, \ldots, x_{p}\right]$ if

$$
\forall s \in\{p+1, \ldots, \operatorname{card}(\mathcal{T})\} x_{s}=\tau(s)
$$

There is a unique $q \in\{p+1, \ldots, \operatorname{card}(\mathcal{T})\}$ such that $\tau(q)=2 \cdot D\left(x_{1}, \ldots, x_{p}\right)$. For each ring $\boldsymbol{K}$ extending $\mathbb{Z}$ the system $\mathcal{H}$ implies $2 \cdot D\left(x_{1}, \ldots, x_{p}\right)=x_{q}$. To see this, we observe that there exist pairwise distinct $t_{0}, \ldots, t_{m} \in \mathcal{T}$ such that $m>p$ and

$$
\begin{gathered}
t_{0}=1 \wedge t_{1}=x_{1} \wedge \ldots \wedge t_{p}=x_{p} \wedge t_{m}=2 \cdot D\left(x_{1}, \ldots, x_{p}\right) \wedge \\
\forall i \in\{p+1, \ldots, m\} \exists j, k \in\{0, \ldots, i-1\}\left(t_{j}+t_{k}=t_{i} \vee t_{i}+t_{k}=t_{j} \vee t_{j} \cdot t_{k}=t_{i}\right)
\end{gathered}
$$

For each ring $\boldsymbol{K}$ extending $\mathbb{Z}$ and for each $x_{1}, \ldots, x_{p} \in \boldsymbol{K}$ there exists a unique tuple $\left(x_{p+1}, \ldots, x_{\operatorname{card}(\mathcal{T})}\right) \in \boldsymbol{K}^{\operatorname{card}(\mathcal{T})-p}$ such that the tuple $\left(x_{1}, \ldots, x_{p}, x_{p+1}, \ldots, x_{\operatorname{card}(\mathcal{T})}\right)$ solves the system $\mathcal{H}$. The sought elements $x_{p+1}, \ldots, x_{\operatorname{card}(\mathcal{T})}$ are given by the formula

$$
\forall s \in\{p+1, \ldots, \operatorname{card}(\mathcal{T})\} \quad x_{s}=\tau(s)\left(x_{1}, \ldots, x_{p}\right)
$$

Lemma 3. The system $\mathcal{H} \cup\left\{x_{q}+x_{q}=x_{q}\right\}$ can be simply computed. For each ring $\boldsymbol{K}$ extending $\mathbb{Z}$, the equation $D\left(x_{1}, \ldots, x_{p}\right)=0$ is equivalent to the system $\mathcal{H} \cup\left\{x_{q}+x_{q}=x_{q}\right\} \subseteq E_{\operatorname{card}(\mathcal{T})}$. Formally, this equivalence can be written as

$$
\begin{gathered}
\forall x_{1}, \ldots, x_{p} \in \boldsymbol{K}\left(D\left(x_{1}, \ldots, x_{p}\right)=0 \Longleftrightarrow \exists x_{p+1}, \ldots, x_{\operatorname{card}(\mathcal{T})} \in \boldsymbol{K}\right. \\
\left.\left(x_{1}, \ldots, x_{p}, x_{p+1}, \ldots, x_{\operatorname{card}(\mathcal{T})}\right) \text { solves the system } \mathcal{H} \cup\left\{x_{q}+x_{q}=x_{q}\right\}\right)
\end{gathered}
$$

For each ring $\boldsymbol{K}$ extending $\mathbb{Z}$ and for each $x_{1}, \ldots, x_{p} \in \boldsymbol{K}$ with $D\left(x_{1}, \ldots, x_{p}\right)=0$ there exists a unique tuple $\left(x_{p+1}, \ldots, x_{\mathrm{card}(\mathcal{T})}\right) \in \boldsymbol{K}^{\mathrm{card}(\mathcal{T})-p}$ such that the tuple $\left(x_{1}, \ldots, x_{p}, x_{p+1}, \ldots, x_{\operatorname{card}(\mathcal{T})}\right)$ solves the system $\mathcal{H} \cup\left\{x_{q}+x_{q}=x_{q}\right\}$. Hence, for each ring $\boldsymbol{K}$ extending $\mathbb{Z}$ the equation $D\left(x_{1}, \ldots, x_{p}\right)=0$ has the same number of solutions as the system $\mathcal{H} \cup\left\{x_{q}+x_{q}=x_{q}\right\}$. 
Putting $M=M / 2$ we obtain new families $\mathcal{T}$ and $\mathcal{H}$. There is a unique $q \in$ $\{1, \ldots, \operatorname{card}(\mathcal{T})\}$ such that

$$
\begin{gathered}
\left(q \in\{1, \ldots, p\} \wedge x_{q}=D\left(x_{1}, \ldots, x_{p}\right)\right) \vee \\
\left(q \in\{p+1, \ldots, \operatorname{card}(\mathcal{T})\} \wedge \tau(q)=D\left(x_{1}, \ldots, x_{p}\right)\right)
\end{gathered}
$$

The new system $\mathcal{H} \cup\left\{x_{q}+x_{q}=x_{q}\right\}$ is equivalent to $D\left(x_{1}, \ldots, x_{p}\right)=0$ and can be simply computed.

It is unknown whether $\mathbb{Z}$ is existentially definable in $\mathbb{Q}$. If it is, then a strong variant of the Bombieri-Lang conjecture is false, see [6, p. 21, Theorem 20].

Theorem 3. (cf. [21] p. 180, Theorem 3.1]) Let $f: \mathbb{N} \rightarrow \mathbb{N}$ be a computable function. If $\mathbb{Z}$ is definable in $\mathbb{Q}$ by an existential formula, then there is a positive integer $q$ and a system $S \subseteq E_{q}$ such that $S$ has infinitely many rational solutions and they all belong to $\mathbb{Q}^{q} \backslash[-f(q), f(q)]^{q}$.

Proof. If $\mathbb{Z}$ is definable in $\mathbb{Q}$ by an existential formula, then $\mathbb{Z}$ is definable in $\mathbb{Q}$ by a Diophantine formula. By Lemma 3 ,

$$
\forall t_{1} \in \mathbb{Q}\left(t_{1} \in \mathbb{Z} \Longleftrightarrow \exists t_{2}, \ldots, t_{p} \in \mathbb{Q} \Phi\left(t_{1}, t_{2}, \ldots, t_{p}\right)\right)
$$

where $\Phi\left(t_{1}, t_{2}, \ldots, t_{p}\right)$ is a conjunction of formulae of the forms $t_{i}=1, t_{i}+t_{j}=t_{k}$, $t_{i} \cdot t_{j}=t_{k}$, where $i, j, k \in\{1, \ldots, p\}$. The function $\mathbb{N} \ni n \rightarrow f(n \cdot p)+1 \in \mathbb{N}$ is computable. By Theorem 1, there is a positive integer $m$ and a system $S \subseteq E_{m}$ such that $S$ has infinitely many integer solutions and they all belong to $\mathbb{Z}^{m} \backslash[-f(m \cdot p), f(m \cdot p)]^{m}$. The following system

$$
\left\{\begin{array}{l}
\text { all equations occurring in } S \\
\text { all equations occurring in } \Phi\left(x_{1}, x_{1,2}, \ldots, x_{1, p}\right) \\
\text { all equations occurring in } \Phi\left(x_{2}, x_{2,2}, \ldots, x_{2, p}\right) \\
\ldots \\
\text { all equations occurring in } \Phi\left(x_{m-1}, x_{m-1,2}, \ldots, x_{m-1, p}\right) \\
\text { all equations occurring in } \Phi\left(x_{m}, x_{m, 2}, \ldots, x_{m, p}\right)
\end{array}\right.
$$

with $m \cdot p$ variables has infinitely many rational solutions and they all belong to $\mathbb{Q}^{m \cdot p} \backslash[-f(m \cdot p), f(m \cdot p)]^{m \cdot p}$. 
For many Diophantine equations we know that the number of integer (rational) solutions is finite, let us recall Siegel's theorem on integral points on curves and Faltings' theorem. Faltings' theorem tell us that certain curves have finitely many rational points, but no known proof gives any bound on the sizes of the numerators and denominators of the coordinates of those points, see [5, p. 722]. In all such cases the Conjecture will allow us to compute such a bound.

Theorem 4. Assuming the Conjecture, if a Diophantine equation $D\left(x_{1}, \ldots, x_{p}\right)=0$ has only finitely many integer solutions, then each such solution $\left(x_{1}, \ldots, x_{p}\right)$ satisfies

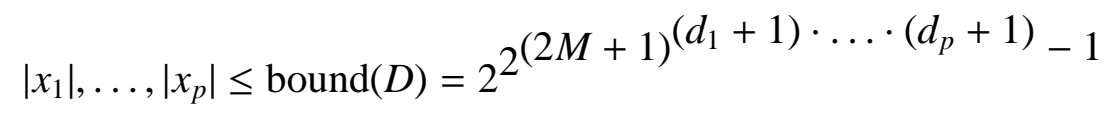

Here, $M$ stands for the maximum of the absolute values of the coefficients of $D\left(x_{1}, \ldots, x_{p}\right), d_{i}$ denote the degree of $D\left(x_{1}, \ldots, x_{p}\right)$ with respect to the variable $x_{i}$.

Proof. It follows from (*) and Lemma 3 ,

Corollary 1. Assuming the Conjecture, for each polynomial $D\left(x_{1}, \ldots, x_{p}\right)$ with integer coefficients

$$
\operatorname{card}\left(\left\{\left(x_{1}, \ldots, x_{p}\right) \in \mathbb{Z}^{p}: D\left(x_{1}, \ldots, x_{p}\right)=0\right\}\right) \in
$$

$$
\left\{0,1,2, \ldots,(1+2 \cdot \operatorname{bound}(D))^{p}\right\} \cup\{\omega\}
$$

Unfortunately, it is undecidable whether a Diophantine equation has infinitely or finitely many solutions in positive integers, see [3]. The same is true when we consider integer solutions or non-negative integer solutions. Moreover, the set of Diophantine equations which have at most finitely many solutions in nonnegative integers is not recursively enumerable, see [16, p. 104, Corollary 1] and [17, p. 240].

For a polynomial $D(x, y)$ with integer coefficients, the following set

$$
\begin{gathered}
\{x \in \mathbb{N}: \exists y \in \mathbb{Z}(D(x, y)=0 \vee D(-x, y)=0)\} \cup \\
\{y \in \mathbb{N}: \exists x \in \mathbb{Z}(D(x, y)=0 \vee D(x,-y)=0)\}
\end{gathered}
$$

consists of non-negative integers. Let $\operatorname{Big}(D)$ denote its supremum in $\mathbb{N}$. Of course, Big : $\mathbb{Z}[x, y] \rightarrow \mathbb{N} \cup\{\infty\}$. Let us consider the following three statements: 
(1) For each polynomial $D(x, y)$ with integer coefficients, it is decidable whether or not the equation $D(x, y)=0$ has only finitely many solutions in integers $x, y$.

(2) The Conjecture.

(3) The function Big is not computable.

Statement (3) expresses the conjecture of J. M. Rojas, see [14], cf. [13]. The negation of statement (3) implies statement (1). By Theorem 4, statements (1)-(3) are jointly inconsistent.

Assuming the Conjecture, if a Diophantine equation has only finitely many integer solutions, then these solutions can be algorithmically found by applying Theorem 4. Of course, only theoretically, because for interesting Diophantine equations the bound $2^{2^{n-1}}$ is too high for the method of exhaustive search. Usually, but not always. The equation $x_{1}^{5}-x_{1}=x_{2}^{2}-x_{2}$ has only finitely many rational solutions $([[11])$, and we know all integer solutions, $(-1,0),(-1,1),(0,0),(0,1)$, $(1,0),(1,1),(2,-5),(2,6),(3,-15),(3,16),(30,-4929),(30,4930)$, see [1]. Always $x_{2}^{2}-x_{2} \geq-\frac{1}{4}$, so $x_{1}>-2$. The system

$$
\left\{\begin{aligned}
x_{1} \cdot x_{1} & =x_{3} \\
x_{3} \cdot x_{3} & =x_{4} \\
x_{1} \cdot x_{4} & =x_{5} \\
x_{1}+x_{6} & =x_{5} \\
x_{2} \cdot x_{2} & =x_{7} \\
x_{2}+x_{6} & =x_{7}
\end{aligned}\right.
$$

is equivalent to $x_{1}^{5}-x_{1}=x_{2}^{2}-x_{2}$. By the Conjecture, $\left|x_{1}^{5}\right|=\left|x_{5}\right| \leq 2^{2^{7-1}}=2^{64}$. Therefore, $\quad-2<x_{1} \leq 2^{\frac{64}{5}}<7132$, so the equivalent equation $4 x_{1}^{5}-4 x_{1}+1=\left(2 x_{2}-1\right)^{2}$ can be solved by a computer.

The algorithm presented in the proof of Lemma 3 is not useful for practical computations, because it introduces a large number of auxiliary variables. Therefore, for the equation $x_{1}^{5}-x_{1}=x_{2}^{2}-x_{2}$ we have chosen the equivalent system which has only 7 variables. In [2, pp. 92-93], M. Cipu studies the system

$$
\left\{\begin{aligned}
x^{2}-3 z^{2} & =1 \\
y^{2}-783 z^{2} & =1
\end{aligned}\right.
$$

for which he constructs various equivalent systems which contain only equations of the forms $x_{i}=1, x_{i}+x_{j}=x_{k}, x_{i} \cdot x_{j}=x_{k}$. 
Assuming the Conjecture, also the heights of rational solutions can be computably bounded from above, as we will show in Theorem 5 .

Lemma 4. ([12] p. 14, the proof of Theorem 1.11]) The integers $A$ and $B>0$ are relatively prime if and only if there exist integers $X$ and $Y$ such that $A \cdot X+B \cdot Y=1$ and $|X| \leq B$.

Theorem 5. Assuming the Conjecture, if a Diophantine equation $D\left(x_{1}, \ldots, x_{p}\right)=0$ has only finitely many rational solutions, then their heights are bounded from above by a computable function of $D$.

Proof. By applying Lemma 3, we can write the equation as an equivalent system $S \subseteq E_{n}$, where $n$ and $S$ can be computed. We substitute $x_{m}=\frac{y_{m}}{z_{m}}$ for $m \in\{1, \ldots, n\}$. Each equation $x_{i}=1 \in S$ we replace by the equation $y_{i}=z_{i}$. Each equation $x_{i}+x_{j}=x_{k} \in S$ we replace by the equation $y_{i} \cdot z_{j} \cdot z_{k}+y_{j} \cdot z_{i} \cdot z_{k}=y_{k} \cdot z_{i} \cdot z_{j}$. Each equation $x_{i} \cdot x_{j}=x_{k} \in S$ we replace by the equation $\left(y_{i} \cdot z_{j} \cdot z_{k}\right) \cdot\left(y_{j} \cdot z_{i} \cdot z_{k}\right)=y_{k} \cdot z_{i} \cdot z_{j}$. Next, we incorporate to $S$ all equations

$$
\begin{aligned}
1+s_{m}^{2}+t_{m}^{2}+u_{m}^{2}+v_{m}^{2} & =z_{m} \\
p_{m} \cdot y_{m}+q_{m} \cdot z_{m} & =1 \\
p_{m}^{2}+a_{m}^{2}+b_{m}^{2}+c_{m}^{2}+d_{m}^{2} & =z_{m}^{2}
\end{aligned}
$$

with $m \in\{1, \ldots, n\}$. By Lagrange's four-square theorem and Lemma 4 , the enlarged system has at most finitely many integer solutions and is equivalent to the original one. Next, we construct a single Diophantine equation equivalent to the enlarged system $S$. For this equation we apply Theorem 4

Theorem 6. Assuming the Conjecture, if a Diophantine equation $D\left(x_{1}, \ldots, x_{p}\right)=0$ has only finitely many solutions in non-negative integers, then the conjectural bound for these solutions can be computed by applying Theorem 4 to the equation

$$
\begin{gathered}
\widehat{D}\left(x_{1}, a_{1}, b_{1}, c_{1}, d_{1}, \ldots, x_{p}, a_{p}, b_{p}, c_{p}, d_{p}\right)= \\
D\left(x_{1}, \ldots, x_{p}\right)^{2}+\left(x_{1}-a_{1}^{2}-b_{1}^{2}-c_{1}^{2}-d_{1}^{2}\right)^{2}+\ldots+\left(x_{p}-a_{p}^{2}-b_{p}^{2}-c_{p}^{2}-d_{p}^{2}\right)^{2}=0
\end{gathered}
$$

Proof. By Lagrange's four-square theorem

$$
\begin{gathered}
\left\{\left(x_{1}, \ldots, x_{p}\right) \in \mathbb{Z}^{p}: \exists a_{1}, b_{1}, c_{1}, d_{1}, \ldots, a_{p}, b_{p}, c_{p}, d_{p} \in \mathbb{Z}\right. \\
\left.\widehat{D}\left(x_{1}, a_{1}, b_{1}, c_{1}, d_{1}, \ldots, x_{p}, a_{p}, b_{p}, c_{p}, d_{p}\right)=0\right\}=
\end{gathered}
$$




$$
\left\{\left(x_{1}, \ldots, x_{p}\right) \in \mathbb{N}^{p}: D\left(x_{1}, \ldots, x_{p}\right)=0\right\}
$$

Since the equation $D\left(x_{1}, \ldots, x_{p}\right)=0$ has only finitely many solutions in nonnegative integers, the equation

$$
\widehat{D}\left(x_{1}, a_{1}, b_{1}, c_{1}, d_{1}, \ldots, x_{p}, a_{p}, b_{p}, c_{p}, d_{p}\right)=0
$$

has only finitely many solutions in integers $x_{1}, a_{1}, b_{1}, c_{1}, d_{1}, \ldots, x_{p}, a_{p}, b_{p}, c_{p}, d_{p}$.

M. Davis, Yu. Matiyasevich and J. Robinson conjecture that there is no algorithm for listing the Diophantine equations with infinitely many solutions, see [4, p. 372].

Theorem 7. The Conjecture implies that the set of Diophantine equations which have infinitely many solutions in integers (non-negative integers) is recursively enumerable.

Proof. The following algorithm works for all polynomials $D\left(x_{1}, \ldots, x_{p}\right) \in \mathbb{Z}\left[x_{1}, \ldots, x_{p}\right]$.

$$
\begin{gathered}
\alpha:=\operatorname{bound}(D)+1 \\
\text { WHILE } \\
D\left(y_{1}, \ldots, y_{p}\right) \neq 0 \text { for all integers } y_{1}, \ldots, y_{p} \text { with } \max \left(\left|y_{1}\right|, \ldots,\left|y_{p}\right|\right)=\alpha \\
\text { DO } \\
\alpha:=\alpha+1
\end{gathered}
$$

Assuming the Conjecture and applying Theorem 4, we conclude that the algorithm terminates if and only if the equation $D\left(x_{1}, \ldots, x_{p}\right)=0$ has infinitely many solutions in integers. For solutions in non-negative integers, we consider the following algorithm:

$$
\begin{gathered}
\theta:=\operatorname{bound}(\widehat{D})+1 \\
\text { WHILE }
\end{gathered}
$$

$D\left(y_{1}, \ldots, y_{p}\right) \neq 0$ for all non-negative integers $y_{1}, \ldots, y_{p}$ with $\max \left(y_{1}, \ldots, y_{p}\right)=\theta$

DO

$$
\theta:=\theta+1
$$

Assuming the Conjecture and applying Theorem 6, we conclude that the algorithm terminates if and only if the equation $D\left(x_{1}, \ldots, x_{p}\right)=0$ has infinitely many solutions in non-negative integers.

Theorem 8. If Matiyasevich's conjecture is true, then for every computable function $f: \mathbb{N} \backslash\{0\} \rightarrow \mathbb{N}$ there is a positive integer $m(f)$ such that for each integer $n \geq m(f)$ there exists a system $S \subseteq E_{n}$ which has only finitely many solutions in integers $x_{1}, \ldots, x_{n}$ and each integer tuple $\left(x_{1}, \ldots, x_{n}\right)$ that solves $S$ satisfies $x_{1}=f(n)+1$. 
Proof. By Matiyasevich's conjecture, the function $\mathbb{N} \backslash\{0\} \ni n \rightarrow f(n)+1 \in \mathbb{N}$ has a finite-fold Diophantine representation. It means that there is a polynomial $W\left(x_{1}, x_{2}, x_{3}, \ldots, x_{r}\right)$ with integer coefficients such that for each non-negative integers $x_{1}, x_{2}$,

$$
\left(x_{2} \geq 1 \wedge x_{1}=f\left(x_{2}\right)+1\right) \Longleftrightarrow \exists x_{3}, \ldots, x_{r} \in \mathbb{N} W\left(x_{1}, x_{2}, x_{3}, \ldots, x_{r}\right)=0
$$

and

only finitely many tuples $\left(x_{3}, \ldots, x_{r}\right) \in \mathbb{N}^{r-2}$ satisfy $W\left(x_{1}, x_{2}, x_{3}, \ldots, x_{r}\right)=0$

By the equivalence (E1) and Lagrange's four-square theorem, for each integers $x_{1}, x_{2}$, the conjunction $\left(x_{2} \geq 1\right) \wedge\left(x_{1}=f\left(x_{2}\right)+1\right)$ holds true if and only if there exist integers

$$
a, b, c, d, \alpha, \beta, \gamma, \delta, x_{3}, x_{3,1}, x_{3,2}, x_{3,3}, x_{3,4}, \ldots, x_{r}, x_{r, 1}, x_{r, 2}, x_{r, 3}, x_{r, 4}
$$

such that

$$
\begin{gathered}
W^{2}\left(x_{1}, x_{2}, x_{3}, \ldots, x_{r}\right)+\left(x_{1}-a^{2}-b^{2}-c^{2}-d^{2}\right)^{2}+\left(x_{2}-\alpha^{2}-\beta^{2}-\gamma^{2}-\delta^{2}\right)^{2}+ \\
\left(x_{3}-x_{3,1}^{2}-x_{3,2}^{2}-x_{3,3}^{2}-x_{3,4}^{2}\right)^{2}+\ldots+\left(x_{r}-x_{r, 1}^{2}-x_{r, 2}^{2}-x_{r, 3}^{2}-x_{r, 4}^{2}\right)^{2}=0
\end{gathered}
$$

The sentence (A) guarantees that for each integers $x_{1}, x_{2}$, only finitely many integer tuples

$$
\left(a, b, c, d, \alpha, \beta, \gamma, \delta, x_{3}, x_{3,1}, x_{3,2}, x_{3,3}, x_{3,4}, \ldots, x_{r}, x_{r, 1}, x_{r, 2}, x_{r, 3}, x_{r, 4}\right)
$$

satisfy the last equality. By Lemma 3 , there is an integer $s \geq 3$ such that for each integers $x_{1}, x_{2}$,

$$
\left(x_{2} \geq 1 \wedge x_{1}=f\left(x_{2}\right)+1\right) \Longleftrightarrow \exists x_{3}, \ldots, x_{s} \in \mathbb{Z} \Psi\left(x_{1}, x_{2}, x_{3}, \ldots, x_{s}\right)
$$

where the formula $\Psi\left(x_{1}, x_{2}, x_{3}, \ldots, x_{s}\right)$ is algorithmically determined as a conjunction of formulae of the forms $x_{i}=1, x_{i}+x_{j}=x_{k}, x_{i} \cdot x_{j}=x_{k}(i, j, k \in\{1, \ldots, s\})$ and for each integers $x_{1}, x_{2}$ at most finitely many integer tuples $\left(x_{3}, \ldots, x_{s}\right)$ satisfy $\Psi\left(x_{1}, x_{2}, x_{3}, \ldots, x_{s}\right)$. Let $m(f)=4+2 s$, and let [·] denote the integer part function. For each integer $n \geq m(f)$,

$$
n-\left[\frac{n}{2}\right]-2-s \geq m(f)-\left[\frac{m(f)}{2}\right]-2-s \geq m(f)-\frac{m(f)}{2}-2-s=0
$$


Let $S$ denote the following system

$$
\left\{\begin{aligned}
\text { all equations occurring in } \Psi\left(x_{1}, x_{2}, x_{3}, \ldots, x_{s}\right) & \\
n-\left[\frac{n}{2}\right]-2-s \text { equations of the form } z_{i}=1 & \\
t_{1} & =1 \\
t_{1}+t_{1} & =t_{2} \\
t_{2}+t_{1} & =t_{3} \\
& \cdots \\
t_{\left[\frac{n}{2}\right]-1}+t_{1} & =t_{\left[\frac{n}{2}\right]} \\
t_{\left[\frac{n}{2}\right]}+t_{\left[\frac{n}{2}\right]} & =w \\
w+y & =x_{2} \\
y+y & =y \text { (if } n \text { is even) } \\
y & =1 \text { (if } n \text { is odd) }
\end{aligned}\right.
$$

with $n$ variables. The system $S$ has only finitely many integer solutions, By the equivalence (E2), $S$ is consistent over $\mathbb{Z}$. If an integer $n$-tuple $\left(x_{1}, x_{2}, x_{3}, \ldots, x_{s}, \ldots, w, y\right)$ solves $S$, then by the equivalence (E2),

$$
x_{1}=f\left(x_{2}\right)+1=f(w+y)+1=f\left(2 \cdot\left[\frac{n}{2}\right]+y\right)+1=f(n)+1
$$

Corollary 2. The Conjecture formulated for an arbitrary computable bound $f: \mathbb{N} \backslash\{0\} \rightarrow \mathbb{N}$ instead of the bound $\mathbb{N} \backslash\{0\} \ni n \rightarrow 2^{2^{n-1}} \in \mathbb{N}$ remains in contradiction to Matiyasevich's conjecture on finite-fold Diophantine representations.

Logicians believe in Matiyasevich's conjecture, but some heuristic argument suggests the opposite possibility. Below is the excerpt from page 135 of the book [15]:

Folklore. If a Diophantine equation has only finitely many solutions then those solutions are small in 'height' when compared to the parameters of the equation. This folklore is, however, only widely believed because of the large amount of experimental evidence which now exists to support it.

Below is the excerpt from page 12 of the article [18]:

Note that if a Diophantine equation is solvable, then we can prove it, since we will eventually find a solution by searching through the countably many possibilities (but we do not know beforehand how far we have to search). So the really hard problem is to prove that there are no solutions when this is the case. A similar 
problem arises when there are finitely many solutions and we want to find them all. In this situation one expects the solutions to be fairly small. So usually it is not so hard to find all solutions; what is difficult is to show that there are no others.

That is, mathematicians are intuitively persuaded that solutions are small when there are finitely many of them. It seems that there is a reason which is common to all the equations. Such a reason might be the Conjecture whose consequences we have already presented.

\section{References}

[1] Y. Bugeaud, M. Mignotte, S. Siksek, M. Stoll, Sz. Tengely, Integral points on hyperelliptic curves, Algebra \& Number Theory 2 (2008), no. 8, 859-885.

[2] M. Cipu, Small solutions to systems of polynomial equations with integer coefficients, An. St. Univ. Ovidius Constanta 19 (2011), no. 2, 89-100, http://www. emis.de/journals/ASUO/mathematics/pdf23/Cipu.pdf, http://www . anstuocmath.ro/mathematics/pdf23/Cipu.pdf.

[3] M. Davis, On the number of solutions of Diophantine equations, Proc. Amer. Math. Soc. 35 (1972), no. 2, 552-554.

[4] M. Davis, Yu. Matiyasevich, J. Robinson, Hilbert's tenth problem. Diophantine equations: positive aspects of a negative solution, in: Mathematical developments arising from Hilbert problems (ed. F. E. Browder), Proc. Sympos. Pure Math., vol. 28, Part 2, Amer. Math. Soc., 1976, 323-378; reprinted in: The collected works of Julia Robinson (ed. S. Feferman), Amer. Math. Soc., 1996, 269-324.

[5] T. Gowers, J. Barrow-Green, I. Leader (eds), The Princeton companion to mathematics, Princeton University Press, Princeton, 2008.

[6] J. Koenigsmann, Defining $\mathbb{Z}$ in $\mathbb{Q}$, http://arxiv.org/abs/1011.3424.

[7] L. B. Kuijer, Creating a diophantine description of a r.e. set and on the complexity of such a description, MSc thesis, Faculty of Mathematics and Natural Sciences, University of Groningen, 2010, http://irs.ub.rug.nl/dbi/4b87adf513823. 
[8] Yu. Matiyasevich, Hilbert's tenth problem, MIT Press, Cambridge, MA, 1993.

[9] Yu. Matiyasevich, Hilbert's tenth problem: what was done and what is to be done. Hilbert's tenth problem: relations with arithmetic and algebraic geometry (Ghent, 1999), 1-47, Contemp. Math. 270, Amer. Math. Soc., Providence, RI, 2000.

[10] Yu. Matiyasevich, Towards finite-fold Diophantine representations, Zap. Nauchn. Sem. S.-Petersburg. Otdel. Mat. Inst. Steklov. (POMI) 377 (2010), 78-90, ftp://ftp.pdmi.ras.ru/pub/publicat/znsl/v377/p078.pdf.

[11] M. Mignotte and A. Pethő, On the Diophantine equation $x^{p}-x=y^{q}-y$, Publ. Mat. 43 (1999), no. 1, 207-216.

[12] W. Narkiewicz, Number theory, World Scientific, Singapore, 1983.

[13] J. M. Rojas, Algebraic geometry over four rings and the frontier to tractability. Hilbert's tenth problem: relations with arithmetic and algebraic geometry (Ghent, 1999), 275-321, Contemp. Math. 270, Amer. Math. Soc., Providence, RI, 2000.

[14] J. M. Rojas, Uncomputably large integral points on algebraic plane curves?, Theoret. Comput. Sci. 235 (2000), no. 1, 145-162.

[15] N. P. Smart, The algorithmic resolution of Diophantine equations, Cambridge University Press, Cambridge, 1998.

[16] C. Smorynski, A note on the number of zeros of polynomials and exponential polynomials, J. Symbolic Logic 42 (1977), no. 1, 99-106.

[17] C. Smorynski, Logical number theory, vol. I, Springer, Berlin, 1991.

[18] M. Stoll, How to Solve a Diophantine Equation, in: An invitation to mathematics: From competitions to research (ed. M. Lackmann and D. Schleicher), Springer, Berlin, 2011, 9-19.

[19] A. Tyszka, Small systems of Diophantine equations which have only very large integer solutions, http://arxiv.org/abs/1102.4122. 
[20] A. Tyszka, Some conjectures on addition and multiplication of complex (real) numbers, Int. Math. Forum 4 (2009), no. 9-12, 521-530, http://www.m-hikari.com/imf-password2009/9-12-2009/tyszkaIMF9-12-2009.pdf.

[21] A. Tyszka, Two conjectures on the arithmetic in $\mathbb{R}$ and $\mathbb{C}$, MLQ Math. Log. Q. 56 (2010), no. 2, 175-184.

Apoloniusz Tyszka

Technical Faculty

Hugo Kołłątaj University

Balicka 116B, 30-149 Kraków, Poland

E-mail address: rttyszka@cyf-kr.edu.pl 